\title{
Probleme der Hörfunkpredigt
}

\author{
von Ambrosius Ruf
}

\section{Die spezifischen Schwierigkeiten des Hörfunkpredigers}

$\mathrm{Zu}$ den grundsätzlichen Lebensvollzügen der Kirche gehört die Verkündigung der frohen Botschaft. So alt die Kirche ist - so lange gibt es in ihr die Predigt, die auf ein je verschiedenes Publikum zugeschnittene - Auslegung und Deutung des Gotteswortes. Die Formen der Verkündigung haben sich im Laufe der Jahrhunderte sehr gewandelt. Man spürt den Unterschied, wenn man etwa die ersten paränetischen Ermahnungen in den Evangelien mit den Homilien der Kirchenväter und diese wiederum mit der barocken Form der Predigt oder der typisch moralischen Predigt der vergangenen Zeit vergleicht. Der inhaltliche Aussagegehalt ist zwar überall der gleiche, jedoch liegt die Spitze der Aussage jeweils anders. - Und dennoch: Vom Urchristentum angefangen bis in die neueste Zeit hinein ist die Predigt ein Bestandteil der Liturgie; sie ist kultischer Vollzug und hat ihren Ort im sakralen Raum, gleichviel ob es sich um die Verkündigung innerhalb der Eucharistiefeier oder um die selbständige Predigt im Gotteshaus (Fastenpredigt und dergleichen) handelt. Neben der ausgesprochen sakralen Verkündigung gab es zwar immer schon die Predigt "auf dem Markt" - die Verkündigung außerhalb des Gotteshauses. Paulus begann damit auf dem Areopag. Franziskus und viele andere führten diese Form der Verkündigung fort. Es darf in diesem Zusammenhang an bedeutende Predigergestalten unserer Zeit erinnert werden, die sich die Verkündigung an die Außenstehenden, also an solche, die nicht mehr zum Gotteshaus kommen, zur Aufgabe gemacht haben. Es scheint, daß die moderne Zeit immer mehr nach Formen der Verkündigung suchen muß, die nicht eine Schar von gläubigen Menschen in einem Gotteshaus anspricht, sondern das breite Publikum auf der Straße.

Eine Möglichkeit zur Verwirklichung dieser "Verkündigung im profanen Raum" ist die Hörfunkpredigt. Sie wird ermöglicht durch eines der jüngsten Kommunikationsmittel und erschließt dieser Form der Verkündigung bisher ungeahnte Möglichkeiten. $\mathrm{Da}$ ist zunächst die Breite der Zuhörerschaft, die angesprochen werden kann. Auch nicht der größte Platz einer Großstadt könnte die Menschen aufnehmen, die morgens einer Hörfunkansprache zuhören. Ein anderes positives Moment kommt dazu: Der Hörer wird in seinem eigenen Milieu angesprochen. Er braucht das Haus nicht zu verlassen. Es bedarf keiner besonderen Propaganda, um ihn zum Zuhören zu bewegen. Die Verkündigung erfolgt in seinen eigenen vier Wänden eine bisher nie dagewesene Möglichkeit. Dadurch kann er in der Anonymität bleiben und muß nicht erst einen eigenen Akt der Bereitschaft zum Zuhören setzen, wie dies immer der Fall ist, wenn er zur Kirche oder zur Predigt auf einen öffentlichen

P. Dr. Ambrosius Karl Ruf OP ist Professor an der Philosophisch-Theologischen Hochschule der Dominikaner zu Walberberg bei Bonn. Er veröffentlichte u. a. "Die Fernsehübertragung der heiligen Messe" (Frankfurt 1964), "Fernsehen, Rundfunk, Christentum“ (Regensburg 21966) sowie im Rahmen seiner Tätigkeit in der katholischen Rundfunkund Fernseharbeit in Deutschland mehrere Fachbeiträge in in- und ausländischen Zeitschriften. 
Platz geht. Das sind zweifellos ungeheure Vorteile der Hörfunkpredigt. Deshalb lohnt es sich, ihre Eigengesetzlichkeit zu studieren und ihnen entsprechend die Verkündigung über den Hörfunk methodisch richtig aufzubauen. Es ist verständlich, daß die Hörfunkpredigt ihren eigenen Gesetzlichkeiten gehorcht, die wiederum von der Eigenart des Mediums abhängen, dessen sie sich bedient. Zunächst soll auf einige Schwierigkeiten hingewiesen werden, denen sich der Hörfunkprediger gegenübersieht:

\section{Die Asynchronität von Aufnahme und Sendung}

Typisch für die Hörfunkpredigt ist es, daß sie - im Gegensatz zu allen bisherigen Formen der Predigt - eine örtliche und meist auch zeitliche Distanz $z$ wischen Prediger und Zuhörer aufweist. Wenn die Predigt nicht unmittelbar (life) übertragen wird, dann muß der Prediger diese doppelte Distanz berücksichtigen: Er sieht und kennt seinen Zuhörerkreis nicht; er spricht seine Ansprache tage- oder wochenlang vor der Sendung auf Band und hat keinerlei direkten Kontakt mit seinen Zuhörern. Daraus entsteht eine Reihe von Schwierigkeiten. Jede Predigt baut an sich auf dem inneren Kontakt zwischen Sprechendem und Hörendem auf. Wie in einem einfachen Gespräch, so ist auch in der Predigt der Redende darauf angewiesen, die Wirkung seiner Worte beim Zuhörer zu spüren, um sich gegebenenfalls korrigieren zu können, um einzelne Punkte nochmals in neuer Formulierung bringen und Unverstandenes besser erklären zu können. Man spricht von einem „virtuellen Dialog“ zwischen Redendem und Hörendem und meint damir jenes Phänomen, das die Aussage immer weiter vorantreibt - bis zu jenem Punkt, an dem die Aussagespitze dem Zuhörer deutlich geworden ist. In diesem Zusammenhang muß auch das erwähnt werden, was man "sensorische Rückwirkungen “ nennt. In jedem Gespräch - auch in der normalen Predigt - muß der Sprechende spüren, wie seine Worte beim Gegenüber „ankommen“. Er muß Zustimmung oder Ablehnung, Kritik oder Begeisterung aus den Zuhörern erspüren, um dementsprechend seine Worte zu formulieren. Bei der Hörfunkpredigt ist das alles nicht möglich, oder doch zumindest sehr viel weniger als bei anderen Formen der Predigt. Der Hörfunkprediger darf nicht der Gefahr verfallen, eine "Vorlesung“ zu halten. Er muß wie jeder Prediger - darauf bedacht sein, daß seine Aussage den Zuhörer "trifft" und ihn in seiner je eigenen Situation anspricht; andernfalls wäre die Hörfunkpredigt bestenfalls ein über den Rundfunk gesendeter religiöser Zeitungsartikel, der in seiner Art wesentlich unverbindlicher ist als das lebendige und gesprochene Wort der Verkündigung. Jeder Prediger vor dem Mikrofon muß sich mit diesem Phänomen auseinandersetzen, - wenn er darauf bedacht ist, daß seine Worte Gehör finden, und es gehört wohl zu den größten Problemen, diese erste Schwierigkeit der Asynchronität von Aufnahme und Sendung zu meistern.

\section{Das differenzierte Publikum}

Die große Chance der Hörfunkpredigt liegt zwar darin, daß sie einen unverhältnismäßig großen Zuhörerkreis anspricht. Hier ist zwar auch gleichzeitig wieder eine neue Schwierigkeit zu sehen: Das Publikum ist differenzierter als jedes andere Publikum bei der Verkündigung im sakralen Raum. Zunächst ist die soziale Differenziertheit zu beachten. Der Publikumskreis des Hörfunkpredigers umfaßt den Akademiker und den einfachen Arbeiter, den Wirtschaftsboß und den Angestellten, den Alten und den Heranwachsenden. Sicher weist auch unser Sonntagskirchenpublikum eine breite Streuung innerhalb der sozialen Berufe auf. Dennoch weiß jeder Prediger meist, wie sein Publikum zusammengesetzt ist. Das gibt ihm leichter die Möglichkeit, 
konkrete Ansätze für seine Aussage zu finden. Das Publikum des Hörfunkpredigers bleibt im Dunkeln. Keiner, der vor dem Mikrofon spricht, weiß, wer letztlich seiner Predigt zuhören wird. Diese Anonymität, die er nicht durchbrechen kann, verführt leicht dazu, die Aussage so allgemein oder abstrakt zu halten, daß sie letztlich niemanden anspricht. Es ist eben schwer, gleichermaßen einen Fabrikdirektor und einen Arbeiter anzusprechen. -

Neben dieser Schwierigkeit ist noch eine andere zu sehen, die noch größer ist: die religiöse Differenziertheit der Zuhörer. Die Verkündigung im sakralen Raum kann darauf aufbauen, daß alle Anwesenden sich zu einer Reihe von Grundsätzen bekennen. Die Zuhörer einer Hörfunkansprache umfassen den religiös Überzeugten genauso wie den Abständigen; die Hörfunkpredigt muß auch den Andersgläubigen und den Ungläubigen berücksichtigen, der meist nur über den Rundfunk ein Bild vom Glauben der anderen Konfession bekommt. Hier ergeben sich zahllose Möglichkeiten, Vorurteile abzubauen und zu einer klareren Vorstellung der Religion oder Konfession zu verhelfen. Das ist zweifellos keine leichte Aufgabe, aber eine sehr lohnende. Auch hier wieder muß der Hörfunkprediger darauf adhten, daß seine Aussage nicht verflacht und er sich nicht auf allgemeine Redensarten beschränkt. Gerade die Hörfunkpredigt sollte ja Abständigen, Andersgläubigen und Ungläubigen die Möglichkeit geben, sich eine klare Vorstellung vom Christentum, seinen Ưberzeugungen und Forderungen zu machen.

\section{Beschränkung der rhetorischen Möglichkeiten}

Jeder Prediger weiß um die Bedeutung des „sermo corporis“. Nicht nur durch seine Stimme, auch durch seine Gesten und durch seine Mimik versucht er seinen Zuhörer zu überzeugen. Die Kunst der Predigt besteht nicht nur darin, wohlgesetzte Worte zu finden. Ein guter Prediger wird darüber hinaus mit den erwähnten sekundären Mitteln der Rhetorik geschickt umzugehen wissen. - Dem Hörfunkprediger sind diese Möglichkeiten genommen. Nur seine Stimme wird gehört. Mit keiner Geste kann er die Bedeutung eines Wortes unterstreichen. Dazu kommt noch, daß selbst seine Stimme beschränkt wird. Das Mikrofon nimmt nur bestimmte Frequenzen auf. Es ist unmöglich, vor dem Mikrofon vom verhalten und leise gesprochenen Wort in das eindringlich fordernde, laute Wort überzugehen.

$\mathrm{Zu}$ dieser - von der Technik bestimmten - Schwierigkeit kommen noch einige andere hinzu, die nur noch am Rande erwähnt werden sollen: die Beschränkung auf die vorgeschriebene Sprechzeit. Sicher wird jeder Prediger versuchen, sich an eine bestimmte Zeit zu halten. Jedoch kommt es normalerweise nicht darauf an, ob er eine Minute länger oder kürzer spricht. Bei einer Hörfunkansprache geht es um Sekunden! Die vorgesehene Sendezeit muß ausgefüllt, darf aber auch nicht überschritten werden. Das verlangt, - und hier liegt die nächste Schwierigkeit, - ein genau auf's Wort ausgearbeitetes Manuskript. Jeder Satz muß genau überlegt sein. Korrekturen während der Aufnahme vor dem Mikrofon sind nicht mehr möglich. Der Prediger ist also fest an sein Manuskript gebunden. Er muß es im Moment der Aufnahme in die Sprechform zurückübertragen. Es darf nicht als "gelesen“ wirken, es muß den Stempel der unmittelbar gesprochenen Ansprache tragen, sonst wird der eigentliche Effekt der Hörfunkpredigt nicht erreicht.

Der Schluß dieses Beitrages mit den Kapiteln „II. Regeln für den Hörfunkprediger" und „III. Verschiedene Formen der Hörfunkpredigt" folgt in Nr. 3 von CS. Dort auch Summary und Resumen. 